\title{
Bacterial Adaptation to Cold
}

\author{
Jaskiran Kaur $^{1 *}$ and Veena Khanna ${ }^{2}$ \\ ${ }^{1}$ Department of Microbiology, ${ }^{2}$ Department of Plant Breeding and Genetics, \\ Punjab Agricultural University, Ludhiana, Punjab, India \\ *Corresponding author
}

\begin{tabular}{|c|c|}
\hline & A B S T R A C T \\
\hline $\begin{array}{l}\text { K e y w o r d s } \\
\text { Bacterial adaptation, } \\
\text { Cold, Temperature. }\end{array}$ & \multirow{3}{*}{$\begin{array}{l}\text { The temperature downshift results in (1) decrease in membrane fluidity (2) } \\
\text { changes in nucleic acid structures (3) reduced ribosome function (4) } \\
\text { inefficient protein folding. Bacteria respond to this low temperature change } \\
\text { by producing cold-shock proteins (CSPs) that bind to single-stranded RNA } \\
\text { and DNA thereby, maintaining cell physiology. CSPs have been widely } \\
\text { studied in Escherichia coli and Bacillus subtilis. Here we discuss the cold } \\
\text { adaptation by desaturase system, RNA chaperone, and transcription } \\
\text { antitermination function of CspA homologues. }\end{array}$} \\
\hline Article Info & \\
\hline $\begin{array}{l}\text { Accepted: } \\
\text { 07 September } 2017 \\
\text { Available Online: } \\
\text { 10 November } 2017\end{array}$ & \\
\hline
\end{tabular}

\section{Introduction}

One of the most frequent stresses faced by living organisms is change in temperature. Fluctuations in temperature have widespread effects on the growth and survival of bacteria, which have therefore developed mechanisms that allow them to adapt to these changes. One such mechanism adopted by bacteria is the cold shock response which consists of a number of adaptive changes ranging from alterations in membrane composition to alterations in the global protein profile of the cell (Phadtare, 2004). The cold shock response of a bacterium like Escherichia coli manifests itself as an acclimation phase during which cell growth completely (but temporarily) stops immediately after the temperature downshift. During this phase, numerous cold shock proteins are produced which help the cells to adopt to low temperature. After the acclimation phase is over, the synthesis of most of the cold shock proteins decreases to a new basal level and synthesis of non-cold shock proteins resumes, allowing cells to grow at low temperature, although at a slower rate (Ermolenko and Makhatadze, 2003).

Some bacteria such as $B$. subtilis and Lactobacillus lactis do not exhibit a growth cessation upon cold-shock response but instead immediately proceed to slow-rate lowtemperature growth (Weber and Marahiel 2003). The chief consequences of temperature downshift include (1) decrease in membrane fluidity affecting membrane-associated cellular functions such as active transport and protein secretion (2) changes in nucleic acid structures (3) reduced ribosome function 
which affects translation especially of noncold-shock proteins and (4) inefficient protein folding. Various mechanisms are adapted by the cell to restore the flexibility of membranes for example, increasing proportion of unsaturated fatty acids (UFAs) (Phadtare and Severinov, 2010).

Temperature drop renders the ribosomes nonfunctional in translation of most cellular mRNAs. However, this effect is not as severe for mRNAs of genes encoding cold-shock proteins. This selective behaviour is due to structural elements present in cold-shock proteins mRNAs that promote translation initiation at low temperature. After the temperature downshift during the lag period, cold-shock ribosomal factors such as RbfA and CsdA are produced. These proteins bind the ribosomes and convert them to coldadapted ribosomes that are capable of translating non-cold-shock mRNAs (Jones and Inouye, 1996).

\section{Structure and function of CSPs}

Cold-induced proteins currently identified belong to the widespread family of small, nucleic acid binding CSPs and represent the prototype of the cold shock domain, which is conserved from bacteria to man (Wolffe et al., 1992). The cold shock domain harbours the nucleic acid binding motifs, RNP-1 and RNP2 (Landsman, 1992). CSPs, with a length of ca. 70 amino acids, share a highly similar overall fold. This fold consists of five antiparallel sheets, forming a $\beta$-barrel structure, with surface exposed aromatic and basic residues (RNP-1 and RNP-2 motifs on strands 2 and 3 , respectively). These residues have been shown to be responsible for the nucleic acid binding properties of this protein family whose members possess variable binding affinities and sequence selectivities (Lopez and Makhatadze, 2000). With the general exception of archaea and cyanobacteria, this protein family has been identified in almost all psychrotrophic, mesophilic, thermophilic and hyperthermophilic bacteria so far examined, and is also present in the earliest diverging bacterial branches Thermotoga and Aquifex which indicates an ancient origin (Graumann and Marahiel, 1998). In B. subtilis, three coldinducible CSPs have been identified (CspBD) while in E. coli only four (CspA, CspB, CspG and CspI) (Wang et al., 1999), out of a total of nine CSPs (CspA-I), are cold induced (Yamanaka 1999).

\section{Role of cold shock proteins in RNA modulation}

The different types of proteins that restructure RNAs are: (1) RNA chaperones that melt misfolded RNA molecules and thus promote proper folding (2) RNA annealers that accelerate annealing of complementary RNAs (3) RNA helicases that resolve the RNA structures using ATP hydrolysis and (4) specific RNA binding proteins that can also contribute to RNA folding by stabilizing specific RNA structures (Rajkowitsch et al., 2007).

\section{Adaptation of the cell membrane}

A rapid temperature downshift can induce phase separation of cell membrane phospholipids, and, as a result, there is a decrease in membrane fluidity and an increase in permeability (Cao-Hoang et al., 2010). The membrane of Gram-negative cells is composed of lipopolysaccharides (LPS), which consist of a distal polysaccharide (Oantigen), and a core polysaccharide and lipid A. E. coli lipid A consists of two glucosamines with attached acyl chains (fatty acids); laurate is the fatty acyl chain usually detected in the cells growing at $37^{\circ} \mathrm{C}$. At low temperatures, there is a decrease in laurate counterbalanced by the appearance of 
palmitoleate (Carty et al., 1999). Effect of low temperature is counteracted by palmitoleate, an unsaturated fatty acid which increases membrane fluidity and lowers phase transition temperature in contrast to laurate. In $E$. coli, the cold-induced acyltransferase LpxP is responsible for attaching palmitoleate to lipid A upon temperature downshift (Vorachek-Warren et al., 2002).

In Bacillus subtilis, adaptation of membrane fluidity involves rapid desaturation of fatty acids in already existing phospholipids. This happens by induction of fatty acid desaturase (Des), regulated by the sensor kinase DesK and the response regulator DesR (Aguilar et al., 2001). The transmembrane domain of DesK acts as sensor of membrane fluidity (Albanesi et al., 2004). A shift to lower temperature causes a decrease in membrane fluidity, which favours the DesK kinase state; DesK phosphorylates the transcriptional activator DesR which subsequently binds to the promoter of the des gene and activates synthesis of the D5-desaturase.This enzyme catalyses the introduction of a double bound into pre-existing fatty acids tails of phospholipids inside the cell membrane (Aguilar et al., 2001; Albanesi et al., 2004).

\section{Regulation at the level of transcription}

The $\operatorname{csp} A$ transcript undergoes a 4-5-fold increase upon cold shock as revealed by studies using reporter gene fusions the reporter genes (Goldenberg et al., 1997; Mitta et al., 1997). No additional factors (other than cold) are necessary for $\operatorname{csp} A$ induction. The elements that enhance the level of $\operatorname{csp} A$ promoter activity include (1) an AT-rich sequence (UP element) immediately upstream of the -35 region (Goldenberg et al., 1997; Mitta et al., 1997) (2) an extended -10 box a TGn motif preceding the -10 box. However, none of these elements seem to specifically contribute to low-temperature activity of the $\operatorname{csp} A$ promoter. The $\operatorname{csp} A, \operatorname{cspB}, \operatorname{cspG}$ and cspI genes possess a long 5' untranslated region (5'-UTR) which contains a highly conserved, 11-base sequence termed the 'cold box'. At high concentration CspA protein binds the cold-box and thus regulates its own expression. The overproduction of 5'UTR leads to prolonged synthesis of CspA, an effect suppressed by co-overproduction of CspA (Jiang et al., 1996; Fang et al., 1998)

Although CspA is also produced at $37^{\circ} \mathrm{C}$ during early exponential growth phase. This induction results from the location of the $\operatorname{csp} A$ gene near the oriC replication origin, which leads to higher gene dosage and higher stability of $\operatorname{cspA}$ mRNA due to lower RNase activity at this stage of growth (Brandi et al., 1998). Production of CspA is observed at $37^{\circ} \mathrm{C}$ upon nutritional upshift (Yamanaka and Inouye 2001).

\section{mRNA stabilization}

The $\operatorname{csp} A$ mRNA is stabilized immediately after cold shock. This stabilization is likely the major factor that leads to dramatic induction of $\mathrm{CspA}$ at low temperature (Phadtare and Severinov, 2005). The 5'-UTR was shown to be responsible for the extreme instability of $\operatorname{csp} A$ mRNA at $37^{\circ} \mathrm{C}$ (a half-life of $12 \mathrm{~s}$ ) and has a positive effect on its stabilization upon cold shock (a half-life of more than 20 min) (Mitta et al., 1997). The $\operatorname{csp} A$ promoter is active at $37^{\circ} \mathrm{C}$, but the steady-state levels of $\operatorname{csp} A$ RNA and CspA synthesis are low at this temperature due to extreme instability of its mRNA. Using enzymatic and chemical probing, it was recently shown that the $\operatorname{csp} A$ mRNA undergoes a temperature-dependent structural rearrangement at low temperature, likely resulting from stabilization of an otherwise thermodynamically unstable folding intermediate. The "low temperature" structure is more efficiently translated and somewhat 
less susceptible to degradation than the $37^{\circ} \mathrm{C}$ structure (Giuliodori et al., 2005).

\section{CspA homologs as transcription antiterminators}

At low temperature stable secondary structures of RNA are formed that interferes with both transcription and translation elongation. CspA homologs act as RNA chaperones due to their ability to 'melt' the secondary structures in nucleic acids and thus facilitating transcription and translation at low temperature. The function of CspA homologs as RNA chaperones promoting transcription antitermination has been studied in considerable detail (Phadtare et al., 2002). Modulation of transcription termination by RNA-binding proteins involves resolving hairpin structures in nascent RNA that can act as transcriptional terminators or pause sites, thus leading to transcript elongation (Stulke 2002). The three-dimensional structures of CspA from E. coli and CspB from B. subtilis have been resolved by X-ray crystallography and NMR-analysis and found to be very similar (Feng et al., 1998). The protein consists of five antiparallel $\beta$-strands ( $\beta 1$ to $\beta 5)$ that form a $\beta$-barrel structure with two $\beta$ sheets. Two RNA-binding motifs, RNP1 and RNP2, are located on the $\beta 2$ and $\beta 3$ strands, respectively. The proteins have an overall negative surface charge with positively charged amino acids surrounding a surfaceexposed aromatic patch. After the initial approach to a RNA molecule through electrostatic attraction and subsequent binding through stacking of the aromatic RNP side chains with RNA bases, further intramolecular or intermolecular base pairing by a segment of RNA bound to a Csp protein is prevented by charge repulsion (Graumann and Marahiel 1998). CspA and its homologs do not exhibit high degree of specificity for their RNA/ DNA substrates (Lopez et al., 2001). The RNA chaperone activity allows the CspA homologs to act as transcription antiterminators and thus aid in cold acclimation of cells (Phadtare et al., 2002)

The genes malE and malK (membrane related functions), mорA and торB (chaperones), $d p s$, katG, rpoS, uspA (stress response) showed firm dependence on Csp proteins for their expression at $15^{\circ} \mathrm{C}$ (Phadtare et al., 2006).

\section{Helicases and Exoribonucleases}

\section{SrmB and CsdA, the DEAD-box helicases involved in cold shock acclimation}

The DEAD-box RNA helicase family proteins play important roles in many cellular processes such as processing, transport or degradation of RNA or ribosome biogenesis (Iost and Dreyfus 2006). The E. coli encodes five DEAD-box RNA helicase family proteins, CsdA, DbpA, RhlB, RhlE and SrmB (Linder et al., 1989) SrmB and CsdA are briefly described below.

\section{SrmB}

$\mathrm{SrmB}$ is involved in ribosome biogenesis. Deletion of $\mathrm{srmB}$ results in (1) slow-growth phenotype at low temperature (2) deficit in free 50S ribosomal subunits and (3) accumulation of a new ribosomal particle sedimenting around 40S. Thus, it was suggested that there is a step of $50 \mathrm{~S}$ assembly, which involves a structural rearrangement that, at least at low temperature, requires SrmB (Charollais et al., 2003).

\section{$\operatorname{CsdA}$}

CsdA has been assigned multiple cellular functions including ribosome biogenesis, translation initiation and degradation of mRNAs. It was shown that CsdA is also involved in the biogenesis of the 50S 
ribosomal subunits (Phadtare et al., 2002) and associates with $50 \mathrm{~S}$ precursors at low temperature (Charollais et al., 2004). CsdA is homologous to eukaryotic translation initiation fac-tor eIF4A. EIF4A catalyzes ATP-dependent unwinding of RNA duplexes and stimulates translational initiation. It was thus suggested that CsdA too may be involved in assisting the translation by promoting translation initiation of structured mRNAs (Lu et al., 1999). The CsdA play a key role in mRNA degradation (1) is found in degradosomes in cold-adapted Cultures (2) is involved in efficient and selective degradation of Csp mRNAs by unwinding the mRNA secondary structure that impedes the processive activity of PNPase (Yamanaka and Inouye 2006). The helicase activity of CsdA is pivotal for promoting the degradation of mRNAs stabilized at low temperature: helicase-deficient CsdA mutants do not complement cold sensitivity of the $\operatorname{csdA}$ deletion cells (Awano et al., 2007)

CsdA and SrmB share several properties: they (1) unwind nucleic acid duplexes with $3^{\prime}$ or $5^{\prime}$ extensions (2) stabilize certain mRNAs (3) bind to RNase E88 (4) participate in 50S assembly probably by modulating RNA structures through their unwinding activity and (5) act as RNA chaperones that prevent misfolding (Iost and Dreyfus 1994)

\section{Cold-inducible exoribonucleases}

\section{RNase R}

PNPase, RNase R and RNase II are the three major 3'-to-5' processing exoribonucleases. These enzymes are primarily involved in RNA metabolism. Both PNPase and RNase R95 are induced by cold shock and are suggested to be the universal degraders of structured RNA in the cell (Cheng and Deutscher 2003). In Bacillus subtilis it was shown that cold-induced helicases and Csps work in together to rescue misfolded mRNA molecules and maintain proper initiation of translation at low temperatures (Hunger et al., 2006)

\section{PNPase}

PNPase is encoded by the pnp gene (Reiner 1969) and its expression is posttranscriptionally autoregulated at the level of both translation and mRNA stability (Jarrige et al., 2001). PNPase binds to the 5' end of RNase III-processed pnp transcript which leads to the inhibition of translation and channeling of $p n p$ mRNA into degradation pathway. During the cold acclimation phase this regulation is temporarily relieved leading to stabilization of the pnp mRNA and thus makes it extremely abundant (Briani et al., 2007). PNPase is one of the main exoribonucleases in the cell and it promotes processive degradation of RNA (Mohanty and Kushner 2003).

\section{Metabolism of RNA at Low Temperature}

At low temperature the RNA metabolism may be different than that at $37^{\circ} \mathrm{C}$ and it requires (1) the assistance of proteins that destabilize RNA secondary structures making them accessible to ribonucleases and (2) coldinducible ribonucleases that can carry out RNA degradation efficiently and selectively to allow cell growth at low temperature. Note that both CsdA and PNPase are essential only at low temperature. Interestingly, these two proteins seem to function independently of each other as they cannot complement each other's functions (Phadtare and Konstantin Severinov 2010).

Temperature is one of the main variable environmental factors potentially impacting viability, and dealing with temperature change is crucial for adaptation. Important changes in RNA metabolism help survival in 
the cold. Cells produce two main groups of cold shock proteins, which modulate the RNA secondary structures and thus play important role(s) in the cold-shock adaptation of cells. Elucidation of the mode of action of various cold shock proteins on each target is necessary for complete understanding of cellular adaptation to low temperatures.

\section{References}

Aguilar P S, Hernandez-Arriaga A M, Cybulski L E, Erazo A C and Mendoza D (2001) Molecular basis of thermosensing: a two-component signal transduction thermometer in Bacillus subtilis. EMBO J 20:1681-91.

Albanesi D, Mansilla M C and Mendoza D (2004). The membrane fluidity sensor DesK of Bacillus subtilis controls the signal decay of its cognate response regulator. J Bacteriol 186: 2655-63.

Awano N, Xu C, Ke H, Inoue K, Inouye M and Phadtare S (2007) Complementation Analysis of the ColdSensitive Phenotype of the Escherichia coli csdA Deletion Strain. Bacteriol 189:5808-15.

Brandi A, Spurio R, Gualerzi C O and Pon C $\mathrm{L}$ (1999) Massive presence of the Escherichia coli ëmajor cold-shock proteiní CspA under non-stress conditions. EMBO J 18:1653-9.

Briani F, Del Favero M, Capizzuto R, Consonni C, Zangrossi S and Greco C (2007) Genetic analysis of polynucleotide phosphorylase structure and functions. Biochimie 89:145-57.

Cao-Hoang L, Dumont F Marechal P A and Gervais P (2010) Inactivation of Escherichia coli and Lactobacillus plantarum in relation to membrane permeabilization due to rapid chilling followed by cold storage. Arch Microbiol 192: 299-305.

Carty S M, Sreekumar K R and Raetz C R
(1999) Effect of cold shock on lipid A biosynthesis in Escherichia coli. Induction at $12 \mathrm{uC}$ of an acyltransferase specific for palmitoleoyl-acyl carrier protein. J Biol Chem 274: 9677-85.

Charollais J, Dreyfus M and Iost I (2004) CsdA, a cold-shock RNA helicase from Escherichia coli, is involved in the biogenesis of $50 \mathrm{~S}$ ribosomal subunit. Nucleic Acids Research 32:2751-9.

Charollais J, Pflieger D, Vinh J, Dreyfus M and Iost I (2003) The DEAD-box RNA helicase $\mathrm{SrmB}$ is involved in the assembly of 50S ribosomal subunits in Escherichia coli. Mol Microbiol 48:1253-65.

Cheng Z F and Deutscher M P (2003) Quality control of ribosomal RNA mediated by polynucleotide phosphorylase and RNase R. Proc Nat Acad Sci USA 100:6388-93.

Ermolenko D $\mathrm{N}$ and Makhatadze G I. Bacterial cold-shock proteins. Cell Mol Life Sci 59:1902-13.

Fang L, Hou Y and Inouye M (1998) Role of the cold-box region in the $5^{\prime}$ untranslated region of the $\operatorname{csp} A$ mRNA in its transient expression at low temperature in Escherichia coli. $J$ Bacteriol 180:90-5.

Feng W, Tejero R, Zimmerman D E, Inouye M and Montelione G T (1998) Solution NMR structure and back-bone dynamics of the major cold-shock protein (CspA) from Escherichia coli: evidence for conformational dynamics in the singlestranded RNA-binding site. Biochemistry 37:10881-96.

Giuliodori A M, Di Pietro F, Marzi S, Masquida $\mathrm{B}$, Wagner $\mathrm{R}$ and Romby $\mathrm{P}$ (2010) The $\operatorname{csp} A$ mRNA is a thermosensor that modulates translation of the cold-shock protein CspA. Mol Cell 37:21-33.

Goldenberg D, Azar I, Oppenheim A B, Brandi A, Pon C L and Gualerzi CO 
(1997) Role of Escherichia coli cspA promoter sequences and adaptation of translational apparatus in the cold shock response. Mol Gen Genet 256:282-90.

Graumann P L and Marahiel M A (1998) A superfamily of proteins containing the cold shock domain. Trends Biochem Sci 23: 286-9.

Hunger K, Beckering C L, Wiegeshoff F, Graumann P L and Marahiel MA (2006) Cold-induced putative DEAD box RNA helicases CshA and CshB are essential for cold adaptation and interact with cold shock protein $\mathrm{B}$ in Bacillus subtilis. J Bacteriol 188:240-8.

Iost I and Dreyfus M (1994) mRNAs can be stabilized by DEAD-box proteins. Nature 372:193-6.

Iost I and Dreyfus M (2006) DEAD-box RNA helicases in Escherichia coli. Nucleic Acids Res 34:4189-97.

Jarrige A C, Mathy N and Portier C (2001) PNPase autocontrols its expression by degrading a double-stranded structure in the pnp mRNA leader. EMBO J 20:6845-55.

Jiang W, Fang L and Inouye M (1996) The role of the 5'-end untranslated region of the mRNA for CspA, the major coldshock protein of Escherichia coli, in cold-shock adaptation. $J$ Bacteriol 178:4919-25.

Jones P G and Inouye M (1996) RbfA, a 30S ribosomal binding factor, is a coldshock protein whose absence triggers the cold-shock response. Mol Microbiol 21:1207-18.

Landsman D (1992) RNP-1, an RNA-binding motif is con-served in the DNA-binding cold shock domain. Nucleic Acids Res 20: 2861-4.

Linder P, Lasko P F, Ashburner M, Leroy P, Nielsen P J and Nishi K (1989) Birth of the D-E-A-D box. Nature 337:121-2.

Lopez M M, Yutani K and Makhatadze G I (1999) Interactions of the major cold shock protein of Bacillus subtilis $\mathrm{CspB}$ with single-stranded DNA templates of different base composition. J Biol Chem 274: 601-8.

Lopez M M, Yutani K and Makhatadze G I (2001) Interactions of the cold shock protein CspB from Bacillus subtilis with single-stranded DNA. Importance of the $\mathrm{T}$ base content and position within the template. J Biol Chem 276:15511-8.

Lu J, Aoki H and Ganoza M C (1999) Molecular characterization of a prokaryotic translation factor homologous to the eukaryotic initiation factor eIF4A. Int J Biochem Cell Biol 31:215-29.

Mitta M, Fang L and Inouye M (1997) Deletion analysis of $\operatorname{csp} A$ of Escherichia coli: requirement of the AT-rich UP element for $\operatorname{csp} A$ transcription and the downstream box in the coding region for its cold shock induction. Mol Microbiol 26:321-35.

Mohanty B K and Kushner S R (2003) Genomic analysis in Escherichia coli demonstrates differential roles for polynucleotide phosphorylase and RNase II in mRNA abundance and decay. Mol Microbiol 50:645-58.

Phadtare S (2004) Recent developments in bacterial coldshock response. Curr Issues Mol Biol 6:125-36.

Phadtare S and Severinov K (2005) Extended -10 motif is critical for activity of the $\operatorname{csp} A$ promoter but does not contribute to low-temperature transcription. $J$ Bacteriol 187:6584-9.

Phadtare S and Severinov K (2010) RNA remodeling and gene regulation by cold shock proteins. RNA Biol 7: 788-95.

Phadtare $S$, Inouye $M$ and Severinov $K$ (2002) The nucleic acid melting activity of Escherichia coli $\mathrm{CspE}$ is critical for transcription antitermination and cold acclimation of cells. $J$ Biol Chem 277:7239-45. 
Phadtare S, Tadigotla V, Shin W H, Sengupta A and Severinov K (2006) Analysis of Escherichia coli global gene expression profiles in response to overexpression and deletion of $\mathrm{CspC}$ and CspE. $J$ Bacteriol 188:2521-7.

Rajkowitsch L, Chen D, Stampfl S, Semrad K, Waldsich C and Mayer O (2007) RNA chaperones, RNA annealers and RNA helicases. RNA Biol 4:118-30.

Reiner A M (1969) Characterization of polynucleotide phosphorylase mutants of Escherichia coli. J Bacteriol 97:1437-43.

Stulke J (2002) Control of transcription termination in bac-teria by RNAbinding proteins that modulate RNA structures. Arch Microbiol 177:433-40.

Vorachek-Warren M K, Carty S M, Lin S, Cotter R J and Raetz C R (2002) An Escherichia coli mutant lacking the cold shock-induced palmitoleoyl transferase of lipid A biosynthesis: absence of unsaturated acyl chains and antibiotic hypersensitivity at $12 \mathrm{uC}$. $\mathrm{J} \mathrm{Biol} \mathrm{Chem}$ 277: 14186-93.

Wang N, Yamanaka K and Inouye M (1999) CspI, the ninth member of the CspA family of Escherichia coli, is induced upon cold shock. J Bacteriol 181: 16039.

Weber M H and Marahiel M A (2003) Bacterial cold shock responses. Sci Prog 86:9-75.

Wolffe A P, Tafuri S, Ranjan M and Familari M (1992) The Y-box factors: a family of nucleic acid binding proteins conserved from Escherichia coli to man. New Biol 4: 290-8.

Yamanaka K (1999) Cold shock response in Escherichia coli. J Mol Microbiol Biotechnol 1: 193-202.

Yamanaka K and Inouye M (2001) Induction of CspA, an E coli major cold-shock protein, upon nutritional upshift at 37 degrees C. Genes Cells 6:279-90.

\section{How to cite this article:}

Jaskiran Kaur and Veena Khanna. 2017. Bacterial Adaptation to Cold. Int.J.Curr.Microbiol.App.Sci. 6(11): 628-635. doi: https://doi.org/10.20546/ijcmas.2017.611.075 